\title{
Histoplasmose cutânea primária: relato de caso em paciente imunocompetente e revisão de literatura
}

\author{
Primary cutaneous histoplasmosis: case report on an \\ immunocompetent patient and review of the literature
}

\author{
Mauricio Naoto Saheki ${ }^{1}$, Armando de Oliveira Schubach ${ }^{1}$, Mariza de Matos Salgueiro', \\ Fátima Conceição-Silva², Bodo Wanke e Márcia Lazera ${ }^{3}$
}

\begin{abstract}
RESUMO
É relatado um caso de histoplasmose cutânea primária em um homem de 45 anos, com apresentação de um nódulo eritematoso no dorso da mão direita acompanhado de linfadenomegalia regional indolor, que se desenvolveu após trauma local ocorrido durante treinamento militar em túnel habitado por morcegos. 0 exame histológico de biópsia da lesão cutânea mostrou um infiltrado granulomatoso, porém não evidenciou elementos fúngicos. 0 cultivo deste material incubado em Ágar Sabouraud mostrou crescimento de Histoplasma capsulatum. Não foi encontrada evidência de envolvimento sistêmico ou imunossupressão. 0 tratamento com 400mg diários de itraconazol oral durante 6 meses resultou na remissão completa da lesão, mantida um ano após o término do tratamento.
\end{abstract}

Palavras-chaves: Histoplasmose cutânea primária. Histoplasma capsulatum. Itraconazol.

\begin{abstract}
This report describes a case of primary cutaneous histoplasmosis in a 45-year-old male. The presentation consisted of an erythematous nodule on the back of the right hand, accompanied by nontender regional lymphadenomegaly that developed following local trauma that occurred during military training in a tunnel inhabited by bats. Histological examination of a biopsy specimen from the skin lesion showed granulomatous infiltrate, but did not show fungal elements. Culturing of this material, incubated in Sabouraud agar, showed growth of Histoplasma capsulatum. No evidence of systemic involvement or immunosuppression was found. Treatment with $400 \mathrm{mg}$ /day of itraconazole orally for six months resulted in complete remission of the lesion, which was maintained one year after the end of the treatment.
\end{abstract}

Key-words: Primary cutaneous histoplasmosis. Histoplasma capsulatum. Itraconazole.

A histoplasmose clássica é causada pelo fungo dimórfico, Histoplasma capsulatum var capsulatum, comumente encontrado em solo contaminado por fezes de aves e morcegos. A doença é endêmica em várias regiões de clima tropical e temperado, sobretudo no continente americano ${ }^{32528}$.

Em áreas endêmicas, as infecções primárias ocorrem tipicamente por inalação de conídios (esporos) suspensos no ar atmosférico de cavernas, galinheiros, construções velhas e abandonadas, locais onde se acumulam excretas de morcegos e/ou aves possibilitando o desenvolvimento saprofítico de Histoplasma capsulatum $^{25} 28$. Em indivíduos imunocompetentes, cerca de 95\% das infecções são assintomáticas, mas ocasionalmente a inalação de conídios pode causar manifestações pulmonares agudas ou crônicas ${ }^{25}$. Em indivíduos imunocomprometidos, particularmente em pacientes com síndrome da imunodeficiência adquirida (SIDA), a histoplasmose pode apresentar-se sob a forma de uma infecção grave, disseminada, como complicação da infecção primária ou reinfecção exógena, ou decorrente da reativação de um foco quiescente, e pode ser fatal em até $80 \%$ dos casos se não tratada ${ }^{15}$.

A lesões cutâneas ocorrem em 4 a $11 \%$ dos pacientes e resultam de invasão secundária da pele em formas disseminadas da infecção $0^{20}$. A histoplasmose cutânea primária (HCP) constitui uma entidade clínica extremamente incomum na literatura. Na maioria dos casos relatados, a lesão ocorreu, provavelmente, por implantação traumática do agente etiológico na pele 1691114172223 .

No presente relato, é apresentado um caso de HCP em paciente imunocompetente e com antecedentes de leishmaniose tegumentar.

\footnotetext{
1. Serviço de Infectologia, Instituto de Pesquisa Clínica Evandro Chagas, Fundação Oswaldo Cruz, Rio de Janeiro, RJ. 2. Departamento de Imunologia, Instituto Oswaldo Cruz, Fundação Oswaldo Cruz, Rio de Janeiro, RJ. 3. Serviço de Micologia, Instituto de Pesquisa Clínica Evandro Chagas, Fundação Oswaldo Cruz, Rio de Janeiro, RJ. Endereço para correspondência: Dr. Mauricio Naoto Saheki. Rua da Lapa 200/sala 908, Centro, 20021-180 Rio de Janeiro, RJ.

Telefax: $55212222-0622$

e-mail: saheki@gmail.com

Recebido para publicação em 13/09/2007

Aceito em 25/11/2008
} 


\section{RELATO DE CASO}

Um homem de 45 anos, branco, militar, natural do Rio Grande do Sul e residente no Rio de Janeiro/RJ, foi atendido no Centro de Referência em Leishmanioses do Instituto de Pesquisa Clínica Evandro Chagas (IPEC/FIOCRUZ) em agosto de 2003 evoluindo há 3 meses com lesão eritemato-pápulo-tuberosa, medindo $1,5 \times 1,0 \mathrm{~cm}$, com vértice ulcerado e recoberto por crostas, localizada no dorso de mão direita, com linfadenomegalia regional associada (Figura 1). 0 exame das mucosas foi normal e não foram encontrados indícios de acometimento pulmonar ou sistêmico.

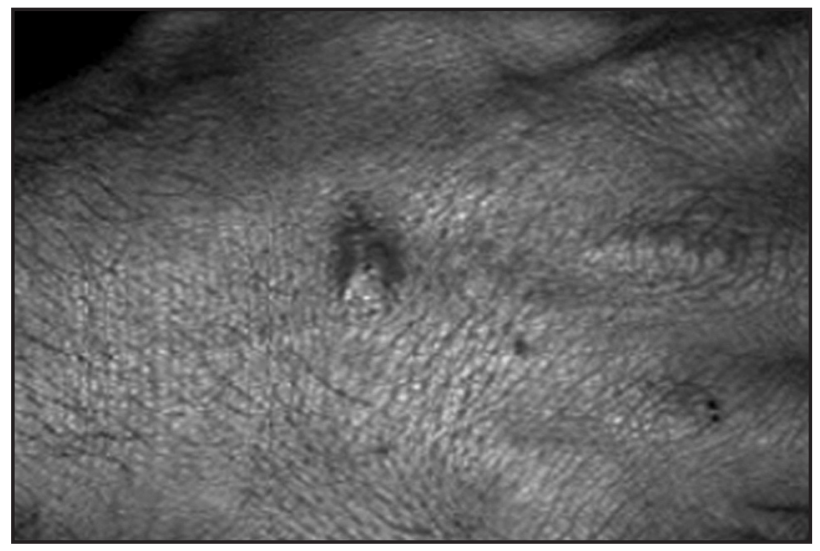

Figura 1 - Foto mostrando lesão eritemato-pápulo-tuberosa.

A lesão cutânea surgiu em maio de 2003, 15 dias após ter rastejado dentro de um túnel infestado por morcegos, com aproximadamente $60 \times 100 \mathrm{~cm}$ de diâmetro e $30 \mathrm{~m}$ de comprimento, em área de instrução militar localizada em Manaus/ AM. Nessa ocasião, sofreu escoriações nos membros superiores, inclusive no local da lesão. Antecedentes: asma na infância, leishmaniose cutânea em face extensora de antebraço direito e mão direita adquirida em Manaus/AM, tratada com antimoniato de meglumina em 1991 e com pentamidina em 1992.

Foi realizada biópsia do bordo da lesão. 0 estudo de cortes histológicos corados pela hematoxilina-eosina (HE) e pelo Grocott demonstrou um processo inflamatório crônico, com diferenciação epitelióide granulomatosa focal, associado a foco de necrobiose, sem evidenciação de fungos ou parasitos. A presença de Histoplasma capsulatum foi evidenciada após cinco semanas de observação dos cultivos do fragmento de lesão em meio ÁgarSabouraud incubados a $25^{\circ} \mathrm{C}$.

A telerradiografia de tórax, de punho direito, e aultrassonografia abdominal foram normais. Os exames rotineiros no sangue (provas de função renal e hepática, glicemia de jejum, eletrólitos, hemograma completo, $\mathrm{LDH}$ ) estavam dentro da normalidade. 0 resultado do teste de Montenegro foi positivo, com induração de 50mm. 0 exame esfregaço por aposição em lâmina corado pelo Giemsa foi negativo para formas amastigotas; o cultivo de material do fragmento de lesão em meio de Novy, Nicolle, McNeal (NNN) mostrou-se negativo. As hemoculturas de sangue periférico foram negativas para bactérias, micobactérias e fungos. Os estudos sorológicos, incluindo pesquisa de anticorpos por imunodifusão para Histoplasma capsulatum e Paracoccidioides brasiliensis, anticorpos para HCV e HBV, VDRL, TPHA, anti-HIV 1 e 2 , e toxoplasmose IgM, foram todos negativos.

Foi iniciado itraconazol $400 \mathrm{mg}$ ao dia por via oral, com remissão completa da lesão após 6 semanas de tratamento, o qual foi mantido até 0 sexto mês. 0 paciente foi acompanhado durante um ano após o término do tratamento, sem sinais ou sintomas de recorrência da doença.

\section{DISCUSSÃO}

Foi realizada uma extensa revisão da literatura, sendo encontrados apenas dezoito casos de HCP. Desde o primeiro caso, descrito por Curtis e Cawley $1947^{8}$, até 1975 , foram relatados seis casos em indivíduos imunocompetentes: dois associados a acidente laboratorial ${ }^{22} 23$, dois sem antecedentes de trauma ${ }^{21}$, um associado a suposto trauma no pé ${ }^{1}$, e uma suposta transmissão venérea ${ }^{17}$. Desde então, os relatos têm sido esporádicos, os mais recentes associados a um estado de imunossupressão: um com papuloeritrodermia ${ }^{13}$, um com artrite reumatóide em terapia imunossupressora ${ }^{14}$, dois com asma brônquica em uso de corticoterapia sistêmica ${ }^{6}{ }^{9}$, um com diabetes mellitus ${ }^{11}$, um com síndrome da imunodeficiência adquirida ${ }^{10}$, e dois em crianças $^{24}$.

As manifestações clínicas foram variáveis e, de modo algum, diagnósticas. A despeito da descrição clássica da HCP como uma lesão única de aspecto cancriforme ${ }^{2223}$, o diagnóstico clínico torna-se difícil por sua natureza polimórfica e inespecífica ${ }^{57}$, confundindo-se com uma variedade de doenças infecciosas e não infecciosas, sobretudo em indivíduos imunocomprometidos ${ }^{10}$ ou após franca exposição ${ }^{19}$. Neste contexto, a confirmação diagnóstica depende principalmente de estudos histopatológicos e da identificação do fungo isolado do tecido excisado ${ }^{5}{ }^{10}$. Em indivíduos imunocompetentes, a infecção costuma ser autolimitada e regredir sem tratamento específico ${ }^{18172122}$. Nesses pacientes, os cultivos teciduais podem estar negativos em mais de $90 \%$ dos $\operatorname{casos}^{1116}$. Devido ao seu curso benigno, a lesão cutânea poderia passar despercebida ou não ser diagnosticada corretamente, o que poderia explicar o pequeno número de casos relatados até 0 momento ${ }^{10} 1123$

Pela similitude do quadro clínico clássico com uma síndrome de inoculação primária, alguns autores ${ }^{22} 23$, propuseram a utilização dos critérios de Wilson para o estabelecimento do diagnóstico de $\mathrm{HCP}^{27}$, os quais são: 1) história de inoculação traumática com desenvolvimento subseqüente de uma lesão cancriforme no período de 3 a 4 semanas no sítio do trauma; 2) isolamento em meio de cultura do fungo causador da lesão; 3) desenvolvimento de linfangite e de linfadenomegalia regional; 4) nenhuma evidência clínica ou laboratorial de infecção sistêmica ou pulmonar prévia e 5) conversão do teste da histoplasmina de negativo para positivo e um título sorológico ascendente. Contudo, através do melhor conhecimento da doença, aliado à descrição de novos casos, têm sido feitas ressalvas à utilização desses critérios, a saber: 1) a lesão cancriforme não é a única apresentação da doença ${ }^{16911141924}$; 2) a lesão primária pode ou não ser acompanhada de linfadenopatia ${ }^{611}$ 14; 3 ) o teste cutâneo da histoplasmina não é mais considerado útil visto que a reação não 
faz distinção entre a infecção atual e a passada, e reações negativas freqüentemente ocorrem em doença disseminada ${ }^{3}$; 4) em pacientes imunodeprimidos, os títulos sorológicos não são confiáveis e decrescem ou desaparecem com a progressão da doença $a^{11} 12$.

0 paciente relatado apresentava uma lesão única associada a uma linfadenomegalia regional sem nenhuma evidência de envolvimento sistêmico ou imunossupressão, da qual foi isolado e identificado Histoplasma capsulatum. 0 estudo histopatológico mostrou a formação de um processo granulomatoso supurativo associado a necrose e infiltrado inflamatório linfocitário e neutrofílico comumente descrito na histoplasmose cutânea.

No presente relato, embora se possa especular sobre a eventual aquisição da infecção por via inalatória seguida de disseminação cutânea progressiva, há fortes argumentos a favor de HCP: 1) a lesão foi adquirida em local altamente suspeito de contaminação por Histoplasma capsulatum e mostrava características próprias de inoculação cutânea primária, 2) não havia sinais ou sintomas que sugerissem foco pulmonar ativo ou cicatrizado, 3) havia história de trauma no local da lesão da qual foi isolado o fungo, 4) não havia evidência atual ou pregressa de envolvimento de órgãos internos. Além disso, o relato anterior de dois outros casos em pacientes imunocompetentes que apresentaram lesões extensas de HCP após visita a uma caverna habitada por morcegos, reforça a idéia de $\mathrm{HCP}^{19}$.

Outro aspecto digno de nota é que diversos surtos de histoplasmose pulmonar aguda têm ocorrido em unidades militares de treinamento em áreas tropicais, mas as manifestações cutâneas não têm sido relatadas.

Ainda no presente caso, é interessante notar que o paciente apresentava concomitantemente positividade no teste de Montenegro, antecedentes de leishmaniose tegumentar e era proveniente de municípios com casos autóctones da doença sendo referenciado ao Instituto de Pesquisas Evandro Chagas por suspeita clínica de leishmaniose. Castro RM e cols ${ }^{4}$ descrevem que a leishmaniose cutâneo-mucosa pode assemelhar-se clinicamente com a histoplasmose mucocutânea, e estudos em militares mostraram que esses são particularmente expostos ao risco de aquisição de leishmaniose, pelo próprio tipo de atividade que desenvolvem, a qual requer contato com matas e a introdução de indivíduos susceptíveis em áreas de endemicidade variável.

Finalmente, cabe mencionar que a maioria dos casos de HCP publicados previamente foram tratados com anfotericina $\mathrm{B}, 0,5 \mathrm{a}$ $0,7 \mathrm{mg} / \mathrm{kg} /$ dia durante 10 a 12 semanas $^{691013}$. Contudo, recentes consensos para o tratamento de histoplasmose em pacientes imunocompetentes recomendam itraconazol nas doses de $200 \mathrm{a}$ $400 \mathrm{mg}$ ao dia durante 6 a 18 meses como terapia de escolha em casos onde não é necessária a internação ${ }^{26}$.

\section{REFERÊNCIAS}

1. Alvarado R, Avendano R, Salfelder K. Primary cutaneous histoplasmosis. Mykosen 19: 259-264, 1976.

2. Butler JC, Heller R, Wright PF. Histoplasmosis during childhood. Southern Medical Journal 87: 476-480, 1994

3. Cano MV, Hajjeh RA. The epidemiology of histoplasmosis: a review. Seminars in Respiratory Infections 16: 109-118, 2001.
4. Castro RM, Pinzón CA, Pássaro EMC, Forjaz MHH, Soler SR. Histoplasmose tegumentar. Anais Brasileiros de Dermatologia 61: 37-40, 1986.

5. Cohen PR, Bank DE, Silvers DN, Grossman ME. Cutaneous lesions of disseminated histoplasmosis in human immunodeficiency virus-infected patients. Journal of the American Academy of Dermatology 23: 422-428, 1990.

6. Cott GR, Smith TW, Hinthorn DR, Liu C. Primary cutaneous histoplasmosis in immunosuppressed patient. Journal of the American Medical Association 242: 456-457, 1979.

7. Couppie P, Clyti E, Nacher M, Aznar C, Sainte-Marie D, Carme B, Pradinaud R. Acquired immunodeficiency syndrome-related oral and/or cutaneous histoplasmosis: a descriptive and comparative study of 21 cases in French Guiana. International Journal of Dermatolology 41: 571-576, 2002.

8. Curtis AC, Cawley EP. Genital histoplasmosis. The Journal of Urology 57: 781-787, 1947.

9. Giessel M, Rau JM. Primary cutaneous histoplasmosis: a new presentation. Cutis 25: 152-154, 1980.

10. Krunic A, Calonje E, Jeftovic D, Berger S, Milinkovic M, Lausevic Z, Martinovic N, Kokai D. Primary localized cutaneous histoplasmosis in a patient with acquired immunodeficiency syndrome. International Journal of Dermatology 34: 558-562, 1995.

11. Krunic AL, Carag H, Medenica MM, Lorincz AL. A case of primary cutaneous histoplasmosis in a patient with diabetes and multi-infarct dementia. Journal of Dermatolology 29: 797-802, 2002.

12. Leimann BC, Pizzini CV, Muniz MM, Albuquerque PC, Monteiro PC, Reis RS, Almeida-Paes R, Lazera MS, Wanke B, Perez MA, Zancope-Oliveira RM. Histoplasmosis in a Brazilian center: clinical forms and laboratory tests. Revista Iberoamericana de Micología 22: 141-146, 2005.

13. Ohnishi K, Nagai Y, Okada K, Ishikawa 0, Miyachi Y. Primary cutaneous histoplasmosis in papuloerythroderma (Ofuji). Journal of Dermatology 21: 586-589, 1994

14. Romano C, Castelli A, Laurini L, Massai L. Primary cutaneous histoplasmosis in an immunosuppressed patient. Mycoses 43: 151-154, 2000.

15. Rubin H, Furcolow ML, Yates JL, Brasher CA. The course and prognosis of histoplasmosis. The American Journal of Medicine 27: 278-288, 1959.

16. Sathapatayavongs B, Batteiger BE, Wheat J, Slama TG, Wass JL. Clinical and laboratory features of disseminated histoplasmosis during two large urban outbreaks. Medicine 62: 263-270, 1983.

17. Sills M, Schwartz A, Weg JG. Conjugal histoplasmosis. A consequence of progressive dissemination in the index case after steroid therapy. Annals of Internal Medicine 79: 221-224, 1973.

18. Singhi MK, Gupta L, Kacchawa D, Gupta D. Disseminated primary cutaneous histoplasmosis successfully treated with itraconazole. Indian Journal of Dermatology, Venereology and Leprology 69: 405-407, 2003.

19. Soo-Hoo TS, Adam BA, Yusof D. Disseminated primary cutaneous histoplasmosis. Australasian Journal of Dermatology 21: 105-107, 1980.

20. Studdard J, Sneed WF, Taylor Jr MR, Campbell GD. Cutaneous histoplasmosis. American Review of Respiratory Disease 113: 689-693, 1976.

21. Symmers WS. Localized cutaneous histoplasmosis. British Medical Journal 2: 790-792, 1956.

22. Tesh RB, Schneidau Jr JD. Primary cutaneous histoplasmosis. The New England Journal of Medicine 275: 597-599, 1966.

23. Tosh FE, Balhuizen J, Yates JL, Brasher CA. Primary Cutaneous Histoplasmosis. Report of a Case. Archives of Internal Medicine 114: 118-119, 1964.

24. Weinberg GA, Kleiman MB, Grosfeld JL, Weber TR, Wheat LJ. Unusual manifestations of histoplasmosis in childhood. Pediatrics 72: 99-105, 1983.

25. Wheat LJ, Freifeld AG, Kleiman MB, Baddley JW, McKinsey DS, Loyd JE, Kauffman CA. Clinical practice guidelines for the management of patients with histoplasmosis: 2007 update by the Infectious Diseases Society of America. Clinical Infectious Diseases 45: 807-825, 2007.

26. Wheat LJ, Kauffman CA. Histoplasmosis. Infectious Disease Clinics of North America 17: 1-19, 2003.

27. Wilson JW, Smith CE, Plunkett OA. Primary cutaneous coccidioidomycosis: the criteria for diagnosis and a report of a case. California Medicine 79: 233-239, 1953.

28. Zancopé-Oliveira RM, Muniz MM, Wanke B. Histoplasmose. In: Coura JR (ed) Dinâmica das doenças infecciosas e parasitárias, Editora Guanabara Koogan, Rio de Janeiro, RJ, p. 1207-1221, 2005. 